\title{
TIMING ANALYSIS AND DESIGN FOR SYSTEM DEVELOPMENT
}

AUTOSAR is established as the standard for development of automotive software-based

systems. The new work package WP-M1 focuses on the practical application of timing analysis

for AUTOSAR-based systems using AUTOSAR artefacts for timing modelling and analysis.

\section{AUTHOR}

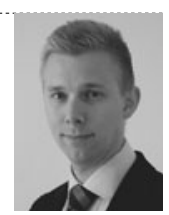

DAN GUNNARSSON is WP-Speaker WP-M1 and works for the BMW Group

in Munich (Germany).

\section{TIMING PROBLEMS IN THE DEVELOPMENT PROCESS}

Since release 4.0, AUTOSAR timing extensions provide the full means necessary to accompany the development process with timing-related information. Mastering the challenges imposed by the increasing technological and organisational complexity, it is highly recommended to support AUTOSAR system development by a systematic approach for timing design and timing analysis. This helps to avoid expensive redesign in later stages of development related to timing problems; applied systematically, it reduces the number of iterations and thus has the potential to shorten the development cycle.

The definition of a corresponding timing methodology is the main goal of the newly established AUTOSAR WP-M1 "Timing analysis". The work package consists of experts and users in the area of timing analysis from AUTOSAR partners with similar problems and heterogeneous tooling working on a topic that is constantly gaining more importance for the automotive industry. In particular it receives support from the AUTOSAR partners: Audi, Bosch, BMW, CEA List, Continental, Daimler, Delphi, General Motors, Gliwa, Inchron, Johnson Controls, Symtavision, Timing Architects, the University of Paderborn, and Volkswagen.

\section{WORK PACKAGE FOCUS}

The activities are concentrated on practical application of timing analysis for AUTOSAR-based systems using AUTOSAR artefacts for timing modelling and analysis. The focus is on typical use cases from the everyday development of complete automotive systems spanning from vehicle networking over Electronic Control Unit (ECU) software integration to code level aspects, $\mathbf{0}$.

For example, a typical use case is to integrate a distributed function like a complex driver assistance system into an existing car. How can this be done efficiently using the AUTOSAR timing models and methodology?

To achieve this, WP-M1 aims to provide a common methodology and defining interaction points for OEMs and suppliers and allow tool vendors to provide the required tooling to fulfil the use cases needed for the development of robust AUTOSAR-based systems. The necessary steps start from high-level functional timing requirements and go down to verification of timing requirements on component (ECU) and system (network) level extending the AUTOSAR methodology and using the AUTOSAR Timex. First public document release is planned for the AUTOSAR release 4.2.1.
Timing analysis and design in the development process

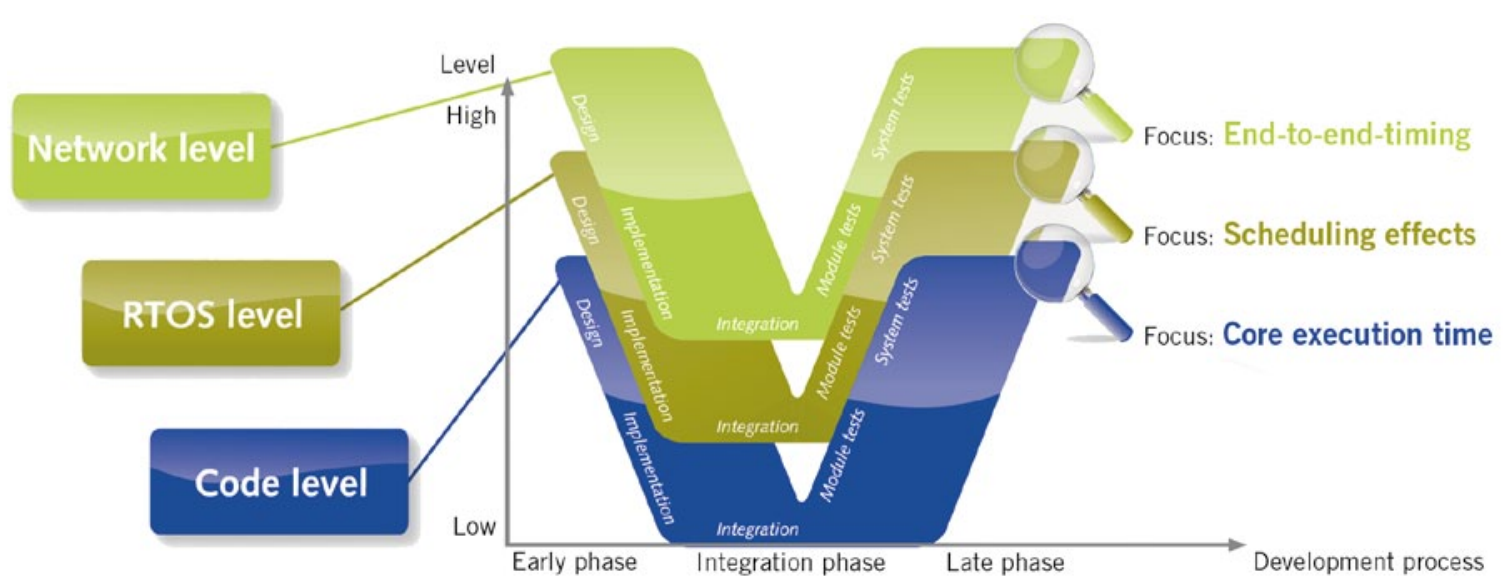

AUTOSAR 